\title{
$\cos -920235-1$
}

\section{Dual Longitudinal Mode Nd:YAG Laser}

T.D. Raymond, A.V. Smith, and P. Esherick

SAND- $-91-2371 \mathrm{C}$

DE92 002012

Sandia National Laboratories

Albuquerque, NM 87185-5800

505-844-9166

\begin{abstract}
We have obtained dual-longitudinal-mode operation of a Q-switched Nd:YAG laser by simultaneously injection-seeding two frequencies to produce pulses with modulation frequency discretely tunable from $185 \mathrm{MHz}$ to greater than $17 \mathrm{GHz}$.
\end{abstract}

\section{DISCLAIMER}

\begin{abstract}
This report was prepared as an account of work sponsored by an agency of the United States Government. Neither the United States Covernment nor any agency thereof, nor any of their employees, makes any warranty, express or implied, or assumes any legal liability or responsibility for the accuracy, completeness, or usefulness of any information, apparatus, product, or rrocess disclosed, or represents that its use would not infringe privately owned rights. Referc..ce tierein to any specific commercial product, process, or service by trade nanie, trademark. manufacturer, or otherwise does not necessarily constitute or imply its endorsement, recommendation, or favoring by the United States Government or any agency thereof. The views and opinions of authors expressed herein do not necessarily state or reflect those of the United States Government or any agency thereof.
\end{abstract}

\section{MASTER}




\title{
Dual Longitudinal Mode Nd:YAG Laser
}

\author{
T.D. R.aymond, A.V. Smith, and P. Esherick \\ Sandia National Laboratories \\ Albuquerque, NM 87185-5800
}

$505-844-9166$

Injection-seeding of pulsed lasers ${ }^{1}$ has proven to be a reliable method of obtaining single-longitudinal-mode (SLM) operation of a high-power oscillator without incurring the high losses associated with intracavity line narrowing elements. Such SLM lasers have found widespread utility in spectroscopic and nonlinear optical measurements. A natural extension of this technology is to simultaneously inject more than one frequency into the power oscillator to generate two or more output frequencies. Such a laser source may find utility in the generation of microwave frequencies via photo-conductive semiconductor switches ${ }^{2}$. Also, the complexity of dual-band differential absorption lidar systems may be reduced by dual-longitudinal-mode (DLM) operation of the power oscillators. In this paper we demonstrate that a Q-switched Nd:YAG laser can be reliably operated on two, independentlyselectable longitudinal modes using commercial components. We show that the two frequencies lead to deeply modulated $1.06-\mu \mathrm{m}$ output pulses having a modulation frequency that can be discretely tuned from $185 \mathrm{MHz}$ to at least $17 \mathrm{GHz}$ in increments of $185 \mathrm{MHz}$ (the slave-cavity free spectral range).

Our experimental layout is shown in Figure 1. The seed oscillators consist of two continuous-wave (cw) SLM Nd:YAG oscillators (Lightwave Electronics Model 102) whose frequencies are conveniently temperature-tuned. The beams from the seed lasers are combined on a $50 \%$ beamsplitter and directed through an optical isolator to the slave oscillator. The remaining $50 \%$ of the combined seed beam is directed to a confocal etalon where we monitor the frequency difference between the seed beams. The power incident on the slave oscillator is approximately $0.4 \mathrm{~mW}$ from each seed laser. The slave oscillator is a Spectra Physics DCR-2A, Q-switched Nd:YAG laser employing a graded-reflectivity output coupler and a rear mirror mounted on a piezo-electric transducer which permits fine cavitylength adjustment. An optional low-finesse intracavity etalon may be inserted into the slavelaser cavity. Injection-seeding is accomplished in the usual fashion ${ }^{3}$ by injecting the slave cavity via the Q-switch polarizer and monitoring the pulse build-up time to optimize the length of the slave cavity. The fundamental and frequency-doubled slave laser output pulses are attenuated and monitored with a fast vacuum photodiode (Hamamatsu R 1328-2) and a 4.5-GHz transient recorder (Tektronics SDC5000). Fixed-gap, etalons of $1-\mathrm{cm}^{-1}$ free spectral range are used to monitor the fundamental and frequency-doubled spectra.

To obtain DLM operation we first optimize the slave cavity length for a single seed laser (seed laser 1 , frequency $\nu_{1}$ ) by monitoring the pulse build-up time. Once this is accomplished, we block seed laser 1 and inject seed laser 2. The frequency of seed laser 2 , $\nu_{2}$, is coarsely set to the desired modulation frequency and then fine adjusted to minimize the cavity build-up time. This procedure sets the frequency detuning between the seed beams, $\delta \nu=\nu_{1}-\nu_{2}$, to be nearly equal to an integral multiple of the slave cavity free spectral range allowing the slave cavity to be simultaneously resonant with both seed frequencies. Once the detuning is adjusted, the slave cavity resonance with both seed frequencies can be maintained in the usual way by minimizing the pulse build-up time. We monitor the build-up time with a detector whose bandwidth is less than $300 \mathrm{MHz}$. For modulation frequencies larger than this, the slave cavity can be locked to the seed beams without additional effort. 
Figure 2 shows the $1.06-\mu \mathrm{m}$ pulse from our slave oscillator when seeded with two seed beams at $\delta \nu=2.4 \mathrm{GHz}$. The modulation clearly demonstrates that the laser is operating on two longitudinal modes separated by 13 cavity resonances. The apparent depth of modulation is limited by the effective bandwidth of the detection system and by imbalance in the intensities at the two output frequencies. The power partitioning between the modes can be adjusted by angle-tuning the intracavity etalon to balance the round-trip gain at the two frequencies. Waveforms at low modulation frequency show nearly $100 \%$ modulation depth indicating that both modes are coaligned to produce much less than one interference fringe over the beam diameter. Since the slave cavity determines both the longitudinal and spatial modes of the output beam, the beat frequency and beam alignment are more tolerant of drif $t$ in the seed frequency and alignment than if the $\mathrm{cw}$ beams were simply pulse amplified. Figure 3 shows the deeply modulated $532-\mathrm{nm}$ output when $\delta \nu=740 \mathrm{MHz}$. To control the phase of the modulation within the pulse envelope we have triggered the Q-switch in phase with the beat from the seed oscillators. Such triggering is particularly important to obtain reproducible waveforms when seeding adjacent modes.

The spectrum of the $1.06-\mu \mathrm{m}$ output, as taken with the $1-\mathrm{cm}^{-1}$ free spectral range etalon, is shown in Fig. 4. The low finesse of this etalon does not permit resolution of the linewidth but, as shown in the top trace, two distinct frequencies are simultaneously produced when both sced beams are injected. The lower two traces show the spectra when only one or the other seed is injected into the slave laser. Note that $\nu_{2}$ is tuned near the gain maximum for Nd:YAG, while $\nu_{1}$ is tuned into the wings of the gain profile. This should be the most stressing case for DLM operation. We have obtained reliable DLM operation when $\nu_{1}$ is detuned from gain center up to at least $17 \mathrm{GHz}$. It should be possible to produce modulation frequencies from $185 \mathrm{MHz}$ to $34 \mathrm{GHz}$, tunable in $185 \mathrm{MHz}$ increments with our present oscillator.

Figure 5 shows the spectrum for the frequency-doubled output. The nonlinear frequency-doubling process generates beams with frequincies $2 \nu_{1}, 2 \nu_{2}$, and $\nu_{1}+\nu_{2}$. The relative ratios of the these three beams should be sensitive to the ratio of power in the two fundamental beams at $\nu_{1}$ and $\nu_{2}$ and hence should be a convenient indicator of the depth of modulation at $1.06 \mu \mathrm{m}$ for large $\delta \nu$ where direct measurement is not practical without a streak camera. A calculation, valid for the low conversion limit, predicts that the intensities at $2 \nu_{1}, \nu_{1}+\nu_{2}$, and $2 \nu_{2}$ should be in the ratio of 1:4:1. The data shown in Fig. 5 were taken with the angle of the frequency-doubling crystal detuned to prevent significant conversion of the $1.06 \mu \mathrm{m}$ beam, but the apparent ratios are not in agreement with this theory.

It should be possible to extend this technique to multiple seeds which could provide a means to control the pulse shape of standard lasers. It may also be useful to operate widely-tunable lasers, such as Ti:Sapphire lasers, to simultaneously access more than one spectral region with a single laser.

1. Y. K. Park, G. Guiliani, and R. L. Byer, Optics Lett. V5, pg. 96 (1980); Y. K. Park, G. Guiliani, and R. L. Byer, IEEE J. Quant. Elect. QE-20, 117 (1984).

2. F. J. Zutavern, G. M. Loubriel, M. W. O'Malley, W. D. Helgeson, and D. L. McLaughlin, in Technical Digest for Conference on Lasers and Electro-Optics, 1991 (Optical Society of America, Washington, D.C., 1991), paper CTuN4.

3. R. L. Schmitt and L. A. Rahn, Applied Optics V25, pg. 629 (1986). 


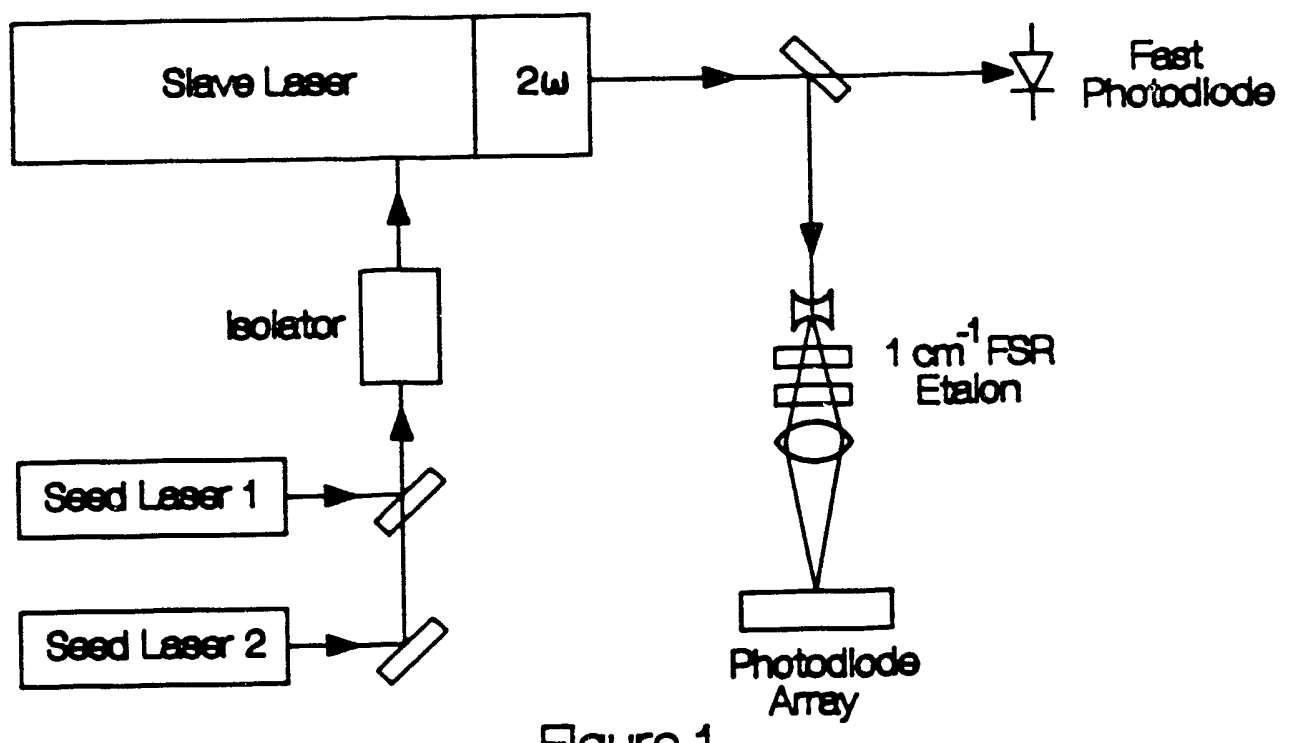

Flgure 1

Figure 2

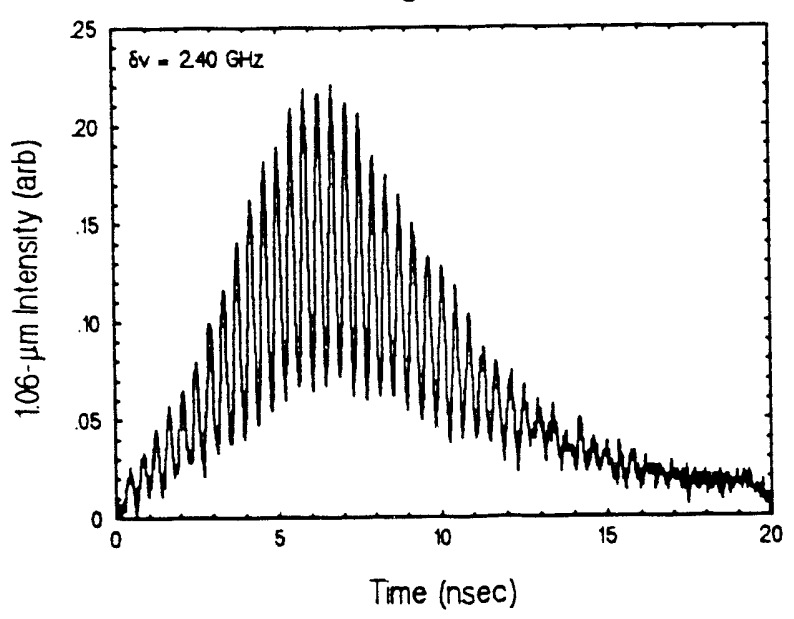

Figure 4

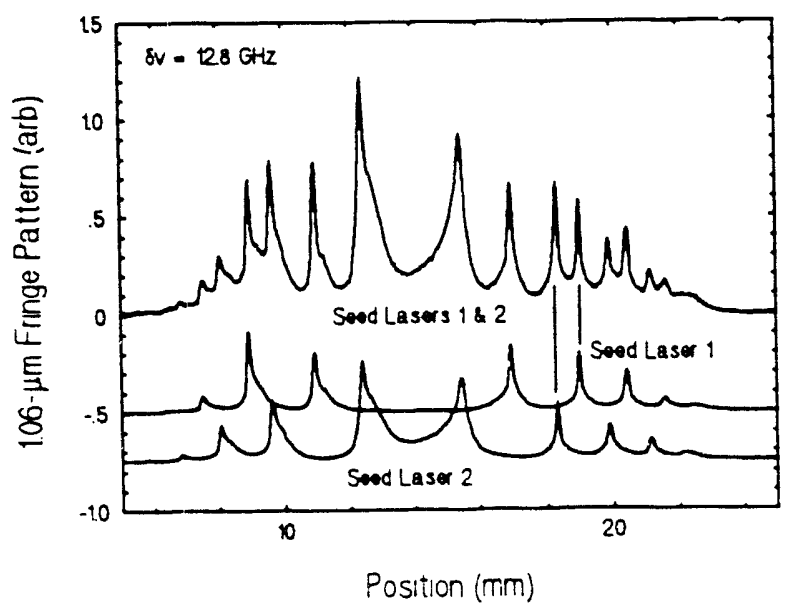

Figure 3

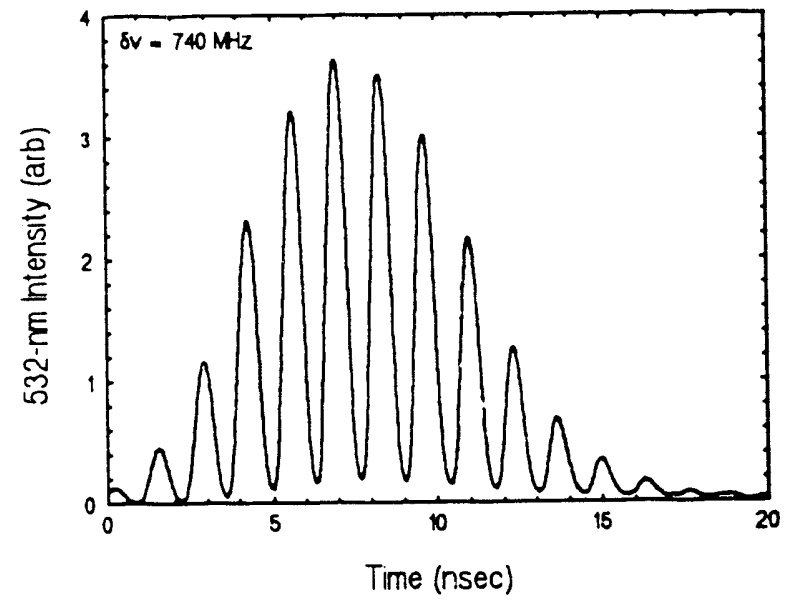

Figure 5

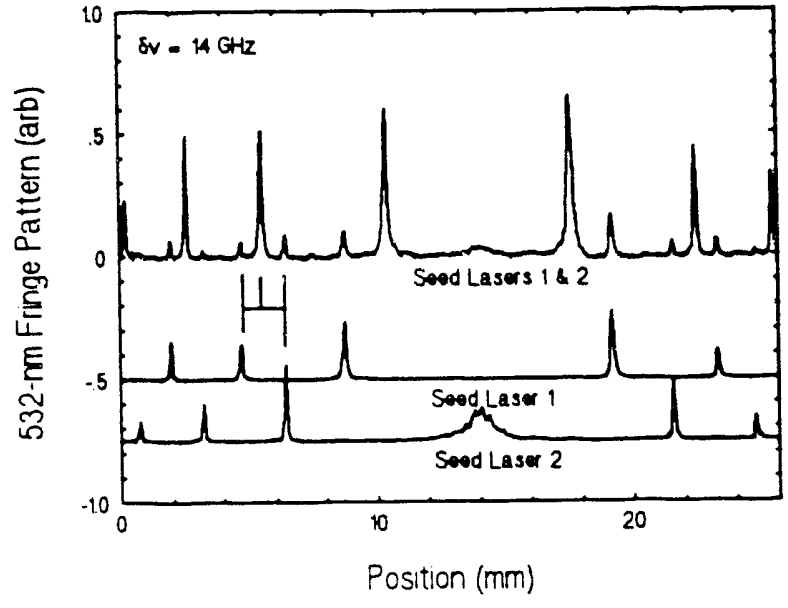



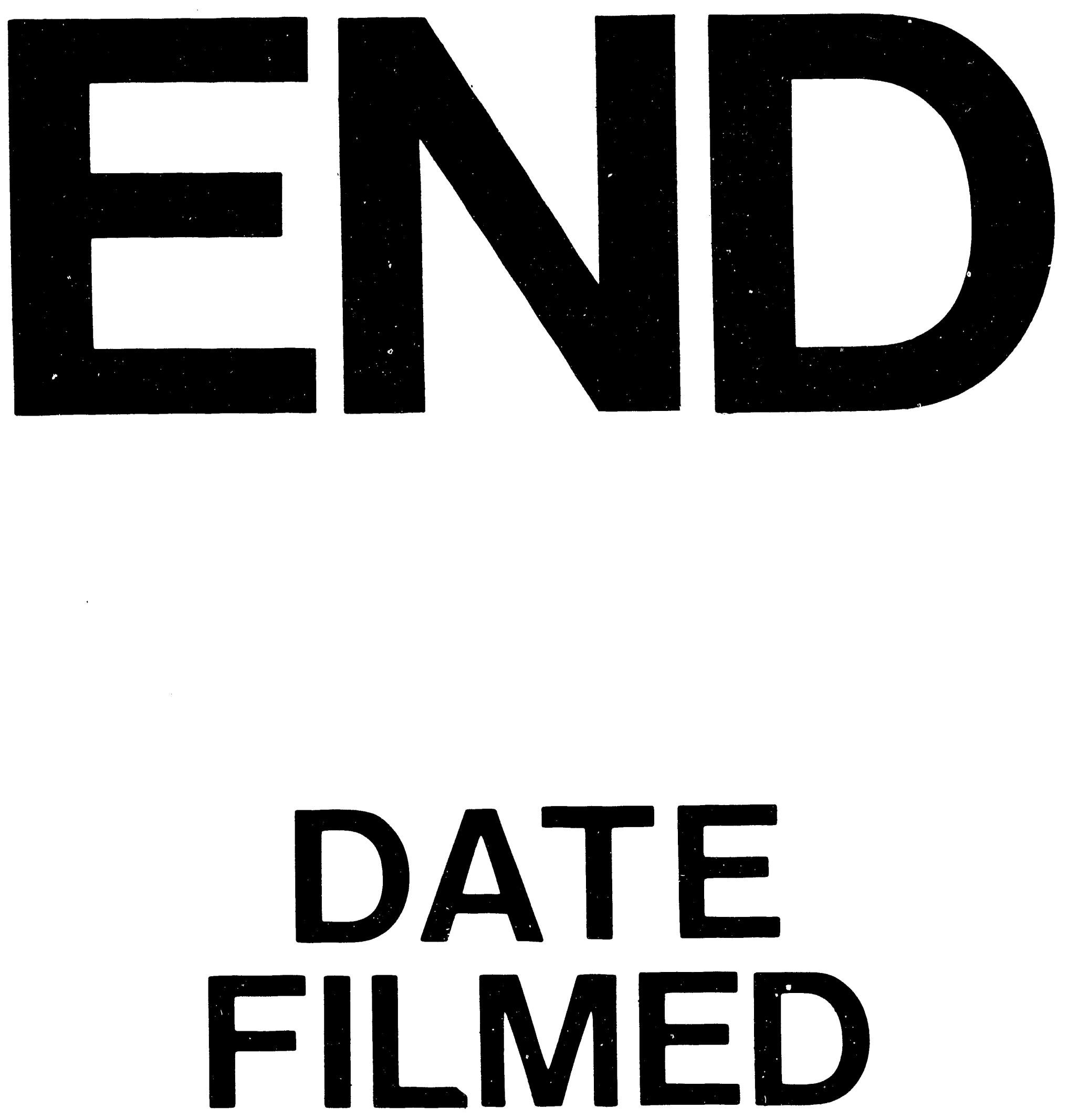

1

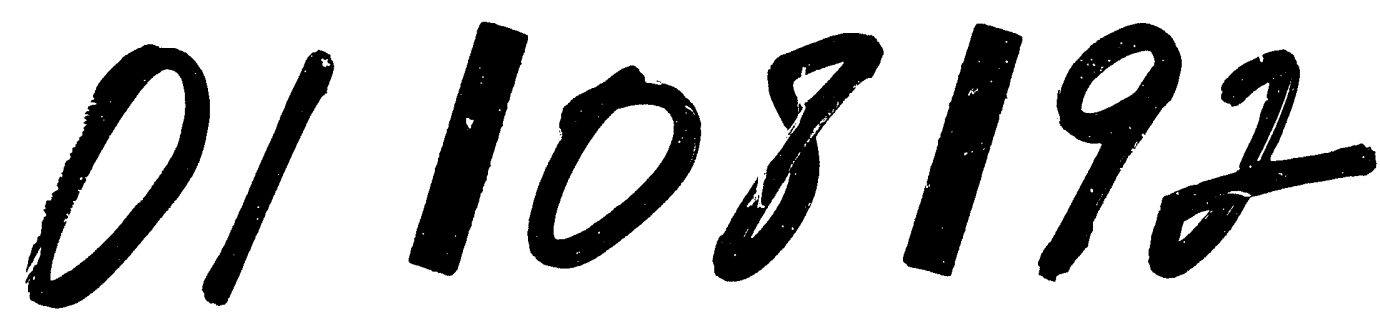


